\title{
Evaluation of Two-Dimensional Phase Unwrapping Algorithms for Interfer- ometric Adaptive Optics Utilizing Liquid-Crystal Spatial Light Modulators
}

\author{
K.L. Baker ${ }^{*}$ and D.A. Silva
}

Lawrence Livermore National Laboratory, Livermore, CA, USA

\begin{abstract}
A Twyman-Green interferometer is used to measure the phase induced by a liquid-crystal spatial light modulator (LC-SLM) via spatial-carrier phase measurement methods in order to characterize the phase response as a function of applied voltage. With the phase response characterized, the LC-SLM can be operated in a closed-loop adaptive optics system. Four separate phase unwrapping algorithms are evaluated for their ability to unwrap the resulting modulo $2 \pi$ signal. The evaluation process consisted of measuring the response of the liquid-crystal spatial light modulator to applied voltages, creating a response matrix for the liquid-crystal spatial light modulator and then running the device in a closed-loop adaptive optics system using the response matrix. The results indicated that the two least-squares phase unwrapping algorithms, based on multigrid techniques and Fourier transform techniques, provided superior performance over a path following algorithm and a two-dimensional extension of Itoh's method when using these algorithms in a closed-loop adaptive optics system.
\end{abstract}

\section{INTRODUCTION}

Numerous devices have been utilized in adaptive optics systems to perform spatial correction of phase aberrations. A partial list of these devices include conventional deformable mirrors, membrane mirrors, MEMS-based spatial light modulators, bimorph mirrors and liquid crystal spatial light modulators [1]. Liquid crystal spatial light modulators have provided the largest actuator count to date, but have had limited utility due to chromatic effects, a low overall phase range limited to approximately a wavelength and speeds ranging from 10 to $30 \mathrm{hz}$. They have, however, been used quite extensively in laboratory testbeds since monochromatic lasers are typically used which eliminates the chromatic effects and phase wrapping can be used to overcome the limited phase range of the devices. Likewise the systems can be scaled such that the correction speed of the spatial light modulator is appropriate for the "simulated" atmospheric turbulence time scale and for the readout speeds of commercial CCD cameras. At that point the advantages of these device, which include their low cost and high spatial correction capability, can be exploited.

In this article we describe a method of characterizing the phase response of a liquid crystal spatial light modulator based on interferometric measurement of the phase applied to the liquid crystal device. Specifically, spatial carrier interferometric techniques are used to determine the phase response modulo $2 \pi$. The wrapped phase must then be unwrapped to determine the phase response. Four separate unwrapping techniques are evaluated as described below [2]. Each of these different techniques is evaluated by running the liquid crystal spatial light modulator in a closed-loop configuration. The residual variance is then used as the metric to determine which techniques provide the best results.

*Address correspondence to this author at the Lawrence Livermore National Laboratory, Livermore, CA, USA; Tel: 925-422-1976; Fax: 925-422-1461; E-mail: baker7@1lnl.gov
In section II, the experimental layout of the phase conjugate engine is presented along with a description of beam propagation through the system. Phase conjugate engine refers to the function of the adaptive optics system to measure the phase aberration in the system and to apply the inverse of the phase or conjugate phase onto the LC-SLM. Section III presents a description of the different phase unwrapping algorithms. Section IV details the experimental results obtained with the different phase unwrapping routines. A discussion on the results is also given in this section. These results are then summarized in the fifth and final section.

\section{EXPERIMENTAL LAYOUT}

The approach taken to perform the initial characterization of the liquid crystal spatial light modulator was to set up a Twyman-Green interferometer as the characterization instrument, as shown in Fig. (1). A tilt is placed on the reference leg of the Twyman-Green interferometer such that a large number of fringes, $\sim 100$, are apparent in the interference pattern on the CCD camera. Fourier transforming the interference pattern produces a Fourier spectrum, which has a DC component and an upper and lower sideband of the two-dimensional Fourier spectrum due to the large carrier frequency introduced by the applied tilt on the reference leg. By filtering all but the upper sideband and shifting this structure back to the origin, the phase can be determined [3]. The wrapped phase calculated in this manner is equal to the inverse tangent of the imaginary part divided by the real part of the inverse Fourier transform. The wrapped phase is then unwrapped using each of the four separate unwrapping schemes. This unwrapped phase is then used to build up a phase response $v s$ applied voltage for each of the actuators on the spatial light modulator.

The LC-SLM is tested in a closed-loop adaptive optics system using each of the unwrapping routines discussed above. The generated phase response $v s$ applied voltage for each of the unwrapping algorithms is evaluated by running the system in a closed-loop adaptive optics system. Closed- 
loop operation refers to the operating mode of an adaptive optics system whereby the deformable mirror surface, the LC-SLM, is measured by the wave-front sensor and the measured wave-front error is driven towards zero phase with subsequent iterations of measurements with the wave-front sensor and corrections applied to the LC-SLM. The carrier frequency interferometer is used as the wave-front sensor to measure the inherent aberrations of the LC-SLM itself. Based on the measured wrapped phase, the phase is then unwrapped and a correction is calculated based on the previously measured phase response vs applied voltage. The correction is then applied to the LC-SLM and a new measurement of the wrapped phase is obtained. This process is then repeated driving the measured aberration on the LC-SLM towards a zero phase.

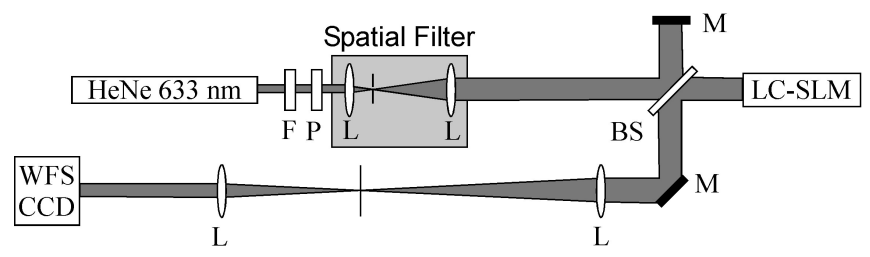

Fig. (1). Twyman-Green interferometer used to characterize the liquid-crystal spatial light modulator. The abbreviations stand for the following: BS, beam splitters; M, mirrors; L, lenses; F, filter; P, polarizer; HeNe, Helium Neon laser; WFS CCD, wave-front sensor charge-coupled device; LCLM, liquid crystal spatial light modulator.

The process of determining the phase response $v s$ applied voltage has been automated and includes the steps described below. The first step is to register the spatial light modulator to the wave-front sensor CCD and remove any rotation between the rows and columns of the liquid crystal spatial light modulator and the CCD camera in software. The registration process is used to determine the projection of the LC-SLM onto the wave-front sensor such that the phase profile measured by the WFS-CCD can be uniquely mapped onto the LCSLM. The initial registration and rotation adjustment are determined by writing three Gaussian phase profiles to three of the corners of the SLM. Interferograms are taken with and without Gaussian phase profiles written to the LC-SLM. The respective phases are unwrapped and the two phases are subtracted to remove the inherent phase aberrations of the unpowered LC-SLM and leave only the three Gaussian phase profiles. A center-of-mass approximation is then used to locate the centers of each of the three Gaussian phase profiles. The rotation is removed by rotating the image acquired from the camera such that the locations of the Gaussian phase profiles, determined by the center-of-mass routines, are along the appropriate row or column in which they were applied to the SLM. The registration process is repeated to verify that the rotation has been removed.

Once the rotation has been removed and the registration performed then a measurement of the phase $v s$ applied voltage can be measured. This is accomplished by sending a series of Gaussian phase profiles of varying phase amplitude to the spatial light modulator. For this process, the Gaussian phase profiles written to the spatial light modulator had a width of 75 pixels, Amp*exp(-(r/width $\left.)^{2}\right)$, and the relative amplitude varied from 0 to 255 in increments of 10 . For each Gaussian a square of 50 by 50 pixels was used, centered around the peak of the Gaussian. By taking a 50x50 pixel region, approximately 81 Gaussian phase profile(9 by 9) locations were written to the spatial light modulator to cover the 440 by 440 pixels characterized on the SLM. For each Gaussian phase profile location, 27 Gaussian phase profiles were written to the LC-SLM with relative amplitudes ranging from 0 to 255 in increments of 10 . Each interferogram recorded was processed to determine the wrapped phase and then the wrapped phase was unwrapped using each of the four different unwrapping routines. The piston and tip/tilt components were removed from the unwrapped phases over regions of the spatial light modulator for which no Gaussian phase was applied. Piston refers to the average phase across the LC-SLM and tip and tilt refer to the average phase slope in $\mathrm{x}$ and $\mathrm{y}$ over the LC-SLM. This was done for the zero phase applied to the LC-SLM as well. These two unwrapped phases, with and without the Gaussian phase profiles added, were subtracted to take out the phase aberration of the unpowered LC-SLM. Once the phase vs applied voltage was determined for each of the unwrapping algorithms, a fourth order polynomial was fit to the response for rapid application of the correction when running in closed-loop.

\section{PHASE UNWRAPPING TECHNIQUES}

Four separate phase unwrapping routines were used to characterize the spatial light modulator and to run the system in a closed-loop adaptive optics test-bed to remove the inherent aberration in the spatial light modulator itself. Two of these algorithms, the multigrid $[4,5]$ and Fourier transform technique $[5,6]$, were based on the method of least squares phase unwrapping. One technique is a path following algorithm [5, 7] and the last technique was based on a twodimensional extension of Itoh's algorithm [5, 8].

One class of techniques for phase unwrapping, mentioned above, is the method of least-squares phase unwrapping. The object of this class of techniques is to minimize the difference between the derivative of the wrapped phase and the unwrapped phase at each of the points in the aperture, the face of the LC-SLM, in the least-squares sense. That implies the minimization of the equation [9]

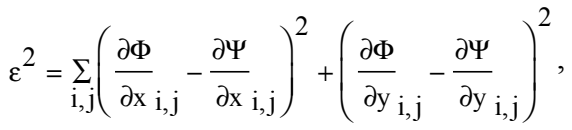

where $\Phi$ represents the unwrapped phase and $\partial \psi / \partial \mathrm{x}$ and $\partial \psi / \partial \mathrm{y}$ represents the measured $\mathrm{x}$ and $\mathrm{y}$ phase derivatives, respectively.

The derivative of the phase is a simple finite difference approximation expressed as $\partial \Phi / \partial \mathrm{x}_{\mathrm{i}, \mathrm{j}}=\Phi_{\mathrm{i}, \mathrm{j}}-\Phi_{\mathrm{i}-1, \mathrm{j}}$ for the $\mathrm{x}$ slope and $\partial \Phi / \partial \mathrm{y}_{\mathrm{i}, \mathrm{j}}=\Phi_{\mathrm{i}, \mathrm{j}}-\Phi_{\mathrm{i}, \mathrm{j}-1}$ for the y slope, where $\partial \Phi / \partial \mathrm{x}_{\mathrm{i}, \mathrm{j}}$ and $\partial \Phi / \partial y_{i, j}$ represent partial derivatives of the phases in the $\mathrm{x}$ and $\mathrm{y}$ directions, respectively. The minimization of Eq. 1 is achieved by taking a derivative of the residual error, $\varepsilon^{2}$, with respect to the reconstructed phase and setting it to zero. The phases in the separate sub-apertures, individual pixels of the WFS-CCD in our case, are assumed to be independent from one another. When these phase derivatives are placed in Eq. 1 above, the minimization of Eq. 1 takes the form 


$$
\begin{array}{r}
\underbrace{2}_{p, q}=0=2 \sum_{i, j}\left(\frac{\partial \Phi}{\partial \mathrm{x}}{ }_{i, j}-\frac{\partial \Psi}{\partial \mathrm{x}}{ }_{i, j}\right)\left(\frac{\partial \Phi_{i+1, j}}{\partial \Phi_{p, q}}-\frac{\partial \Phi_{i, j}}{\partial \Phi_{p, q}}\right)+ \\
\left(\frac{\partial \Phi}{\partial \mathrm{y}_{\mathrm{i}, \mathrm{j}}}-\frac{\partial \Psi}{\partial \mathrm{y}_{\mathrm{i}, \mathrm{j}}}\right)\left(\frac{\partial \Phi_{i, j+1}}{\partial \Phi_{p, q}}-\frac{\partial \Phi_{i, j}}{\partial \Phi_{p, q}}\right)
\end{array}
$$

Because of the assumption of independence, there is only a contribution from the derivatives when the two phases are from the same sub-aperture. This can be expressed in terms of delta functions, allowing Eq. 2 to be rewritten as

$$
\begin{aligned}
& \frac{\partial \varepsilon^{2}}{\partial \Phi}=0=2 \sum_{i, j}\left(\frac{\partial \Phi}{\partial x_{i, j}}-\frac{\partial \Psi}{\partial x_{i, j}}\right)\left(\delta_{p, i+1} \delta_{q, j}-\delta_{p, i} \delta_{q, j}\right) \\
& \partial \Phi \\
& p, q \\
& +\left({\frac{\partial \Phi}{\partial y_{i, j}}}-\frac{\partial \Psi}{\partial y_{i, j}}\right)\left(\delta_{p, i} \delta_{q, j+1}-\delta_{p, i} \delta_{q, j}\right)
\end{aligned}
$$

Eq. 3 can be reduced to a simpler form by replacing the partial phase derivatives in Eq. 3 with their simple finite difference approximation, defined above, and multiplying the delta functions through the equation. After grouping similar functions and recognizing the simple finite derivative of the Laplacian function, $\nabla^{2}$, the following equation is arrived at for phase reconstruction

$$
\frac{\partial \varepsilon^{2}}{\partial \Phi_{p, q}}=0=2 \sum_{\mathrm{p}, \mathrm{q}}\left(\nabla^{2} \Phi_{\mathrm{p}, \mathrm{q}}-\nabla \cdot\left(\nabla \Psi_{\mathrm{p}, \mathrm{q}}\right)\right)
$$

This simply represents the Poisson equation, $\nabla^{2} \Phi=\rho$, with $\rho$ equal to the divergence of the measured phase derivatives.

The boundary conditions for the phase reconstruction problem, using a least-squares wave-front unwrapping method, are derived via a mirror reflection of the phase, $\Phi[5$, $6]$. The phase is defined on the interval $0 \leq \mathrm{i} \leq \mathrm{p}-1$ and $0 \leq \mathrm{j}$ $\leq \mathrm{q}-1$. The phase is reflected about the line $\mathrm{i}=\mathrm{p}-1$ (right edge) and the subsequent phase profile, which is now defined on the interval $0 \leq \mathrm{i} \leq 2 \mathrm{p}-2$ and $0 \leq \mathrm{j} \leq \mathrm{q}-1$, is reflected about the line $\mathrm{j}=\mathrm{q}-1$. This creates a periodic function in which the boundary conditions for the phase derivative are readily derived $[6,5]$ :

$$
\begin{aligned}
& {\frac{\partial \Phi}{\partial x_{-1, j}}}=-{\frac{\partial \Phi}{\partial x_{0, j}}}^{\partial \Phi} \\
& {\frac{\partial \Phi}{\partial x_{p-1, j}}}=-{\frac{\partial \Phi}{\partial x_{p-2, j}}}^{\partial \Phi} \\
& {\frac{\partial \Phi}{\partial y_{i,-1}}}=-{\frac{\partial \Phi}{\partial y_{i, 0}}} \\
& {\frac{\partial \Phi}{\partial y_{i, q-1}}}=-{\frac{\partial \Phi}{\partial y_{i, q-2}}}
\end{aligned}
$$

The phase is still solved on the original grid $0 \leq \mathrm{i} \leq \mathrm{p}-1$ and $0 \leq \mathrm{j} \leq \mathrm{q}-1$. The mirror reflection is simply used to pro- vide the appropriate boundary conditions to ensure that the correct solution is reached.

One of the least squares techniques compared in this article is the multigrid technique $[4,5]$. Iterative techniques such as Gauss elimination, Gauss-Seidel relaxation and simultaneous over-relaxation are very efficient at quickly solving for high spatial frequencies, phase unwrapping high spatial frequencies for the application at hand. These techniques are quite slow in obtaining low spatial frequencies and require many iterations to do so. The multigrid approach solves the Poisson equation derived above on multiple grids, each with a different spatial scale, transferring the residual error between the grids. This allows low spatial frequencies on the finest grid to be transformed into high spatial frequencies on the coarsest grid where they can be solved quickly using the above mentioned techniques such as Gauss-Seidel relaxation. These "high spatial frequencies" on the coarsest grid are then transformed back into low spatial frequencies on the finest grid. This allows multigrid techniques to solve problems very quickly for both the low and high spatial frequencies.

The second least-squares technique compared in this article is one that solves the Poisson equation using Fourier transform techniques $[5,6]$. This technique performs a mirror reflection of the wrapped phase, creating a periodic function that can be solved using fast Fourier transform techniques. It solves the same Poisson "type" equation with a mirror reflection of the phase to set the boundary conditions as the multigrid technique discussed above.

The third technique is a path following technique [5, 7]. This method locates all of the residues in the wrapped phase by summing wrapped phase differences of four adjacent pixels. If the sum is $\pm 2 \pi$, then the pixel is identified as a residue. Branch cuts are generated between the oppositely charged residues. The last step involves a path integration around the branch cuts to determine the unwrapped phase

The fourth technique is the two dimensional extension of Itoh's algorithm $[5,8]$. In this algorithm the derivative of the phase is determined by subtracting the phase from a spatially shifted version of itself. The phase differences are then wrapped modulo $2 \pi$ and a value of a given pixel is set to zero. The phase along that line is then found by summing the wrapped phase differences. The two dimensional unwrapping is found by applying this procedure along one given row in the array to unwrap the phase in that row. Next each column is unwrapped with this procedure, using the value found in the unwrapped horizontal row as the starting point for the unwrapping along each of the columns.

\section{EXPERIMENTAL RESULTS}

The phase response of the liquid-crystal spatial light modulator was determined as discussed above in section II. A series of Gaussian phase profiles were written to the spatial light modulator and an interferogram was recorded for each of these Gaussian phase profiles. The wrapped phase was determined from these interferograms using the procedure discussed above and then unwrapped using the four separate algorithms. The unwrapped phase was subtracted from the zero amplitude Gaussian representing the unpowered surface figure of the spatial light modulator itself to determine the response of the applied phase. A polynomial 
fit of the phase $v s$ amplitude for each of the pixels was then taken. Fig. (2) shows a graph of a single pixel in the spatial light modulator showing the measured phase $v s$ amplitude at each of the 27 amplitudes sent to the spatial light modulator along with the polynomial fit to the data. In this figure the filled black circles represent the amplitude of the Gaussians applied to the spatial light modulator and the corresponding phase measured along the abscissa. The solid gray line corresponds to the polynomial fit to the data used in closed-loop operation of the adaptive optics system. The data depicted in Fig. (2) resulted from an analysis using the unweighted multigrid unwrapping algorithm.

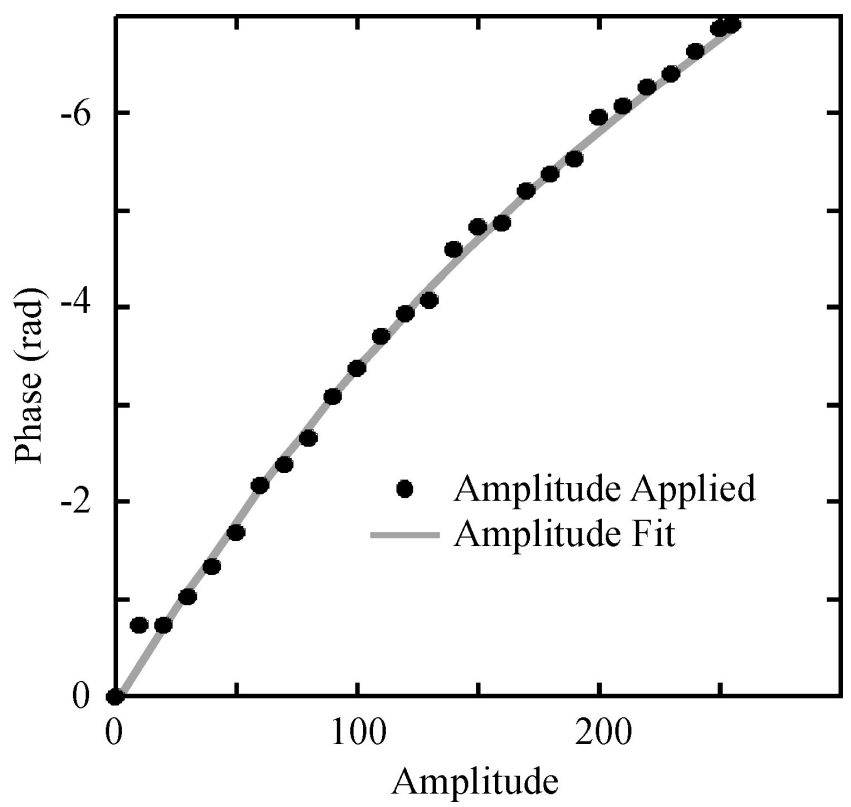

Fig. (2). Measured phase $v s$ amplitude for a single pixel in the spatial light modulator. The experimental data collected and analyzed is shown by the filled black circles and a polynomial fit to the data is represented by the solid gray line.

Given the measured phase vs amplitude response for the liquid crystal spatial light modulator, the next step was to test this response by running the spatial light modulator in closed-loop to take out the residual phase aberration of the unpowered spatial light modulator. The initial phase aberration of the spatial light modulator that was to be removed is shown below in Fig. (3). There is approximately eight radians of phase error peak to valley with the main component being astigmatism. The phase profile in Fig. (3) was reconstructed using the unweighted multigrid unwrapping algorithm.

The unpowered phase aberration of the spatial light modulator was measured using each of the separate unwrapping algorithms. The measured phase profile, determined after unwrapping by the appropriate algorithm, was multiplied by the gain, 0.9 for all cases. The correction for the spatial light modulator was then determined using polynomial fit of the amplitude $v s$ phase tables generated for each of the unwrapping algorithms and applied to the spatial light modulator. The initial correction sent to the spatial light modulator is shown in Fig. (4). An eight bit signal, 0 to 255, was sent to the spatial light modulator to correct the unpowered surface figure error. The residual phase of the corrected

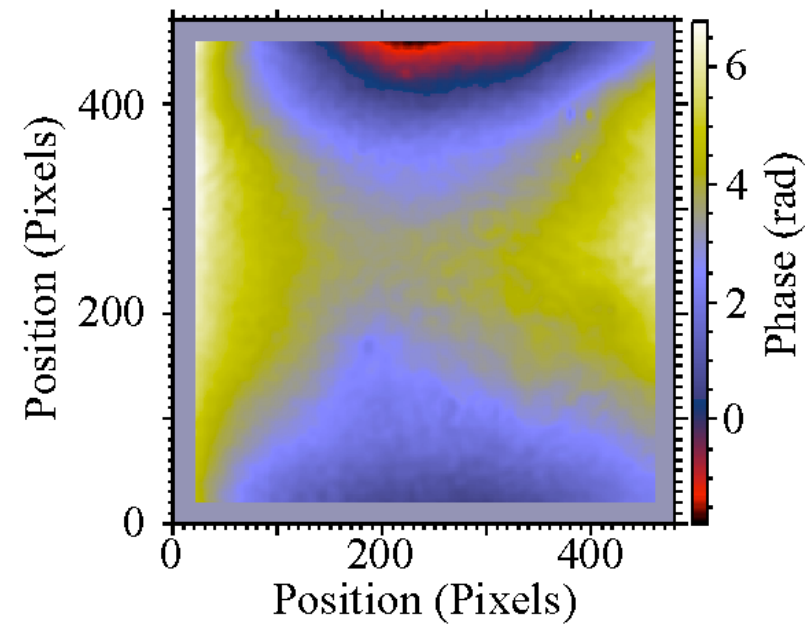

Fig. (3). Unpowered surface figure error of the spatial light modulator

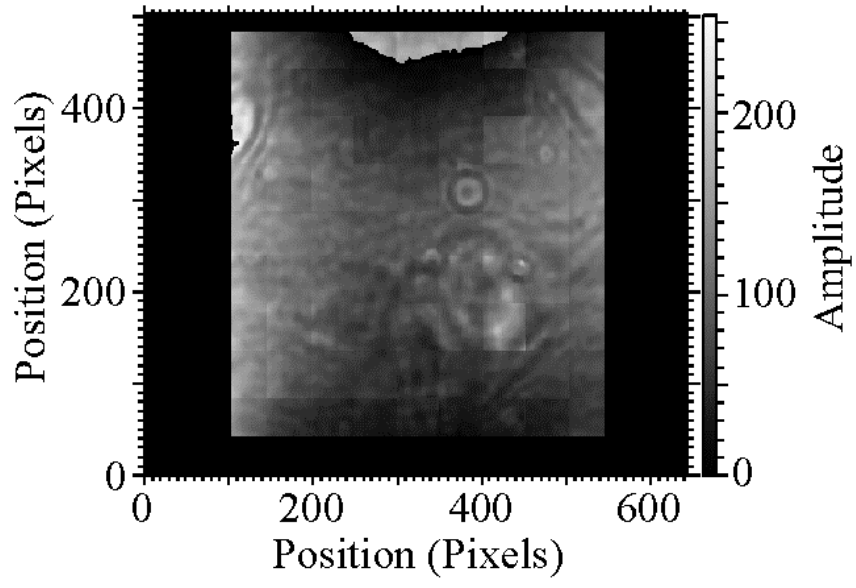

Fig. (4). Amplitude signal sent to the spatial light modulator to correct the unpowered surface figure error in the device.

spatial light modulator is then shown in Fig. (5). It is clear from Fig. (5) that the surface figure error on the spatial light

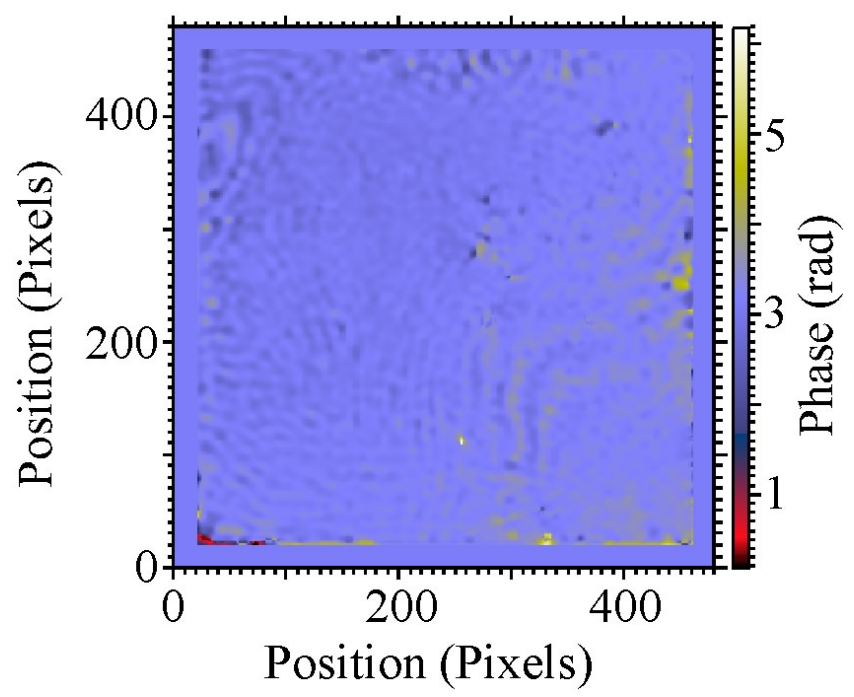

Fig. (5). Residual surface figure error of the spatial light modulator after correction. 
modulator has been significantly reduced. Again this figure was generated using the unweighted multigrid algorithm. Each of the four separate algorithms was used to flatten the spatial light modulator. The results of each of the algorithms are shown in Fig. (6). In particular, Fig. (6) shows the measured residual variance as a function of iteration number for the four separate unfolding algorithms. The solid black line represents the unweighted multigrid algorithm, the dashed gray line corresponds to the Fourier transform unwrapping algorithm, the dashed black line shows the path following algorithm and the solid gray line depicts the two-dimensional extension of Itoh's method. The best performance was obtained using the unweighted multigrid and the Fourier transform algorithms. Fig. (7) represents data taken using only the multigrid unwrapping algorithm. In this case a residual variance, $\sigma^{2}$, of $0.09 \mathrm{rad}^{2}$ was obtained after 10 iterations, as depicted in Fig. (6) and again in Fig. (7a). This corresponds to a Strehl ratio, $S_{r}$, of 0.91 using the "extended" Marechal approximation [1], $S_{\mathrm{r}}=\exp \left(-\sigma^{2}\right)$, as shown in Fig. (7b).

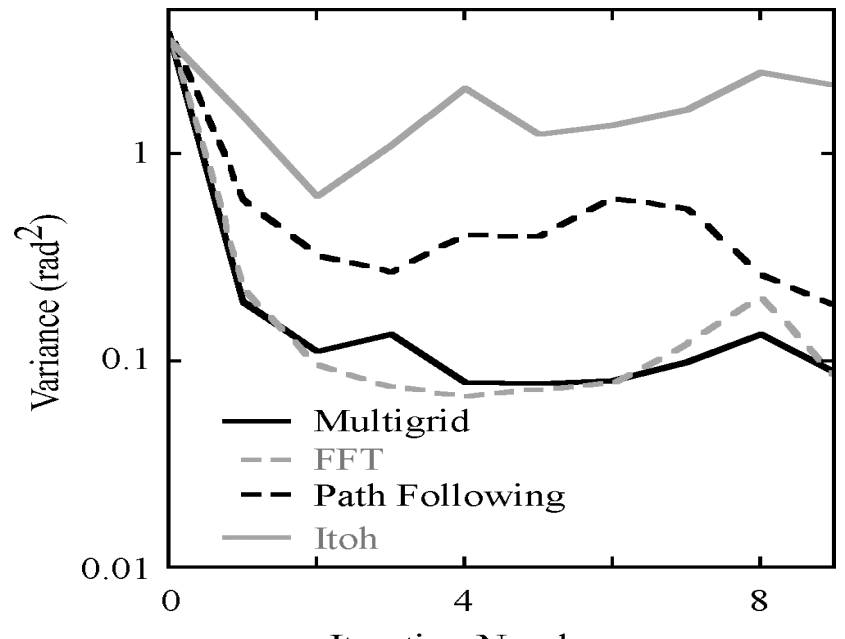

Iteration Number

Fig. (6). The measured variance as a function of iteration number for each of the four unwrapping algorithms. The solid black line represents the unweighted multigrid algorithm, the dashed gray line corresponds to the Fourier transform unwrapping algorithm, the dashed black line shows the path following algorithm and the solid gray line depicts the two-dimensional extension of Itoh's method.
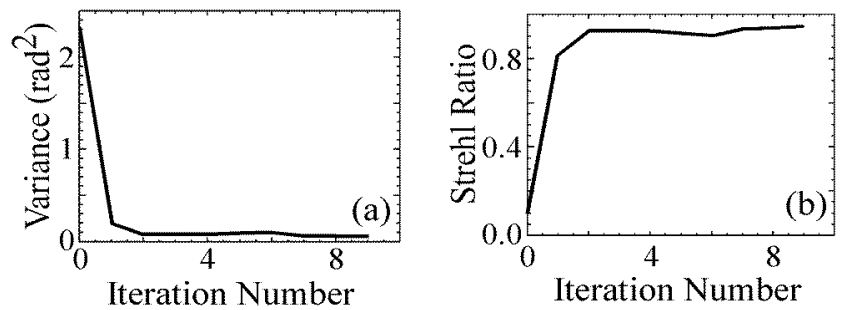

Fig. (7). The measured variance (a) and corresponding Strehl ratio (b) calculated from the Marechal approximation utilizing the multigrid unwrapping algorithm.

\section{SUMMARY}

This article has presented the results of evaluating four phase unwrapping algorithms in the context of their ability to operate a liquid-crystal spatial light modulator in closed-loop operation using an interferometric wave-front sensor. The evaluation process consisted of measuring the response of the liquid-crystal spatial light modulator to applied phase profiles, which required the use of the separate phase unwrapping algorithms. From that measurement a response matrix for the liquid-crystal spatial light modulator was created containing the amplitude $v s$ phase information for each of the pixels and for each of the unwrapping algorithms, as shown in Fig. (2). The liquid-crystal spatial light modulator was then operated in closed-loop with each of the different phase unwrapping algorithms to determine the achievable variance with each of the algorithms. The variance results, shown in Fig. (6), indicate that the two least-squares phase unwrapping algorithms, based on multigrid techniques and Fourier transform techniques, provided superior performance over a path following algorithm and a two-dimensional extension of Itoh's method. Using the multigrid unwrapping algorithm, residual phase variances of 0.09 were achieved in closed-loop which, using the "extended" Marechal approximation, corresponds to a Strehl ratio of 0.91 .

\section{ACKNOWLEDGEMENTS}

The authors would like to thank J.S. Werner for the loan of a Hamamatsu liquid-crystal spatial light modulator. This work was performed under the auspices of the U.S. Department of Energy by Lawrence Livermore National Laboratory in part under Contract W-7405-Eng-48 and in part under Contract DE-AC52-07NA27344.

\section{REFERENCES}

[1] Hardy JW. Adaptive optics for astronomical telescopes. Oxford: Oxford University Press; 1998.

[2] Strand J, Taxt T. Performance evaluation of two-dimensional phase unwrapping algorithms. Appl Opt 1999; 38: 4333-44.

[3] Robinson DW, Reid GT, Eds. Interferogram Analysis. Bristol and Philadelphia: Institute of Physics Publishing; 1993; pp. 167-78.

[4] Pritt MD. Phase unwrapping by means of multigrid techniques for interferometric SAR. IEEE Trans Geosc Remote Sens 1996; 34: 728-38.

[5] Ghiglia DC, Pritt MD. Two-dimensional phase unwrapping. New York: John Wiley \& Sons, Inc.; 1998.

[6] Pritt MD, Shipman JS. Least-squares two-dimensional phase unwrapping using FFT's. IEEE Trans Geosc Remote Sens 1994; 32: 706-8.

[7] Goldstein RM, Zebker HA, Werner CL. Satellite radar interferometry: two-dimensional phase unwrapping. Radio Sci 1988; 23: 71320 .

[8] Itoh K. Analysis of the phase unwrapping algorithm. Appl Opt 1982; 21: 2470.

[9] Fried DL. Least-square fitting a wave-front distortion estimate to an array of phase-difference measurements. J Opt Soc Am 1977; 67: $370-75$. 